and may contribute to behavioural states of depressed mood and anxiety.

\section{'Integrative Neuroscience' and Psychiatry: Identifying Cognitive, Affective and Brainwave Markers of Psychiatric Disorder}

\section{Williams}

The Brain Dynamics Centre, Westmead Millennium Institute, Westmead Hospital and Discipline of Psychological Medicine, University of Sydney, Australia

\section{Overview}

Multidisciplinary efforts have begun to encourage a freer exchange of information - resulting in a more 'integrative neuroscience' across disciplines and theoretical models in psychiatry. This symposium outlines the potential insights into major psychiatric disorders from the first entirely standardized and centralized database, which brings together cognitive, affective, brain function and genetic measures. It contains 5000 healthy subjects $(6-100$ years) and growing psychiatric groups. With these multimodal and standardized data sets, we have identified objective markers that distinguish each disorder and that predict real-life functional outcomes. In this symposium, we outline markers for first-episode schizophrenia, attention deficit hyperactivity disorder, depression and Alzheimer's dementia.

$11-01$

\section{Identifying cognitive, affective and neural synchrony markers which predict real-world functional outcome in first-episode schizophrenia: an integrative neuroscience approach}

\section{Williams' ${ }^{1}$, J Whitford', BJ Liddell ${ }^{1,2}$, D Alexander ${ }^{1,2}$, G Flynn ${ }^{1,3}$, W Wong ${ }^{1,3}$, P Das ${ }^{1,4}$, AWF Harris', E Gordon ${ }^{1,2}$}

'The Brain Dynamics Centre, Westmead Millennium Institute, Westmead Hospital and Western Clinical School, University of Sydney, Australia; ${ }^{2}$ Brain Resource International Database, Brain Resource Company; ${ }^{3}$ Early Psychosis Intervention Program, Liverpool Hospital; and ${ }^{4}$ Neuroscience Institute of Schizophrenia and Allied Disorders (NISAD), New South Wales, Australia

Background: In addition to psychotic symptoms, first-episode schizophrenia (FES) is characterized by profound difficulties in cognition and social and emotional functions.

We examined whether cognitive markers predict realworld functional outcome in FES and whether this prediction is enhanced by social-cognitive markers. We also examined the relationship between these markers and lack of neural synchrony in FES, in the context of our integrative neuroscience model of impaired neural coordination of salient and task-relevant information in this condition.

Method: We tested patients with FES (within 3 months of service contact) using the standardized Brain Resource International Database cognitive battery and tests of social and emotional function (including DASS, NEO-FFI). Neural synchrony was extracted from EEG recorded to corresponding tasks. Assessments of symptoms and functional outcome included PANSS, SOFAS, and World Health Organisation Quality of Life Scale.

Results: General cognitive markers predicted negative symptom severity, with the greatest contribution from poor verbal function, then visuospatial executive functions. Poorer executive function also predicted poorer social and occupational outcome and quality of life. This predictive relationship was significantly improved by the addition of the social-cognitive marker, excessive negativity bias. These combined markers were also related to an excess of high-frequency neural synchrony in EEG recordings.

Conclusion: These findings show that 1) markers of general cognitive dysfunction predict real-world functional outcome in FES, 2) predictive power is enhanced by taking social and emotional aspects of cognition into account and 3) impairments in neural binding and coordination of salient stimuli may underlie these functional difficulties.

\section{$11-02$}

\section{Identifying affective markers within an integrative neuroscience model of depression}

\author{
D Mathersul', A Kemp ${ }^{1,2}$, P Hopkinson ${ }^{1,3}$, E Gordon ${ }^{1,3}$ \\ 'The Brain Dynamics Centre, Westmead Millennium Institute, Westmead Hospital \\ and Western Clinical School, University of Sydney, Australia; ${ }^{2}$ Psychological \\ Medicine, University of Sydney; and ${ }^{3} B r a i n$ Resource International Database, \\ Brain Resource Company, Sydney, Australia
}

Background: Our integrative model of depression focuses on disturbances in affective and cognitive function, which contribute to clinical depression. Endophenotypes for depression include disturbances 